\title{
Digital Wideband Integrators With Matching Phase and Arbitrarily Accurate Magnitude Response
}

\author{
Cagatay Candan
}

\begin{abstract}
A new class of linear-phase infinite-impulse-response digital wideband integrators based on the numerical integration rules is presented. The proposed class of integrators exactly matches the desired phase response of the continuous-time integrator (after group delay compensation) and can approximate the magnitude response as closely as desired by increasing the number of system zeros, i.e., the order of the integrator. The low-order integrators (up to the fourth degree) generated by this technique can be immediately utilized in many applications such as strapdown inertial navigation systems, sampled data systems, and other applications that require accurate integration.
\end{abstract}

Index Terms-Digital integrators, Lagrange interpolation, Newton-Cotes, numerical integration, quadrature.

\section{INTRODUCTION}

$\mathbf{T}$ HE DIGITAL integrators are utilized in many applications including sampled data systems, navigation, and control applications. In this brief, we present a new class of discrete-time infinite-impulse-response filters whose frequency response approximates the frequency response of the continuous-time integrator as accurately as it is desired, i.e.,

$$
H\left(e^{j \omega}\right) \approx \frac{1}{j \omega}=D\left(e^{j \omega}\right)
$$

where $-\pi<\omega<\pi$.

In the equation previously shown, $H\left(e^{j \omega}\right)$ denotes the digital integrator, and $D\left(e^{j \omega}\right)$ denotes the desired response.

Several digital integrator designs have been proposed in the literature [2]-[8]. Among these, the zero-order hold ${ }^{1}$ $H(z)=1 /\left(1-z^{-1}\right)$ and the trapezoidal rule $H(z)=(1+$ $\left.z^{-1}\right) /\left(2\left(1-z^{-1}\right)\right)$ are the simplest and the most well known approximations to the desired response. It can be easily observed that, as $\omega \rightarrow 0$, response $H\left(e^{j \omega}\right) \rightarrow 1 / j \omega$ for both approximations. These simplest approximations can be sufficient for the integration of oversampled signals (signals sampled much above the Nyquist rate), but a closer inspection shows that there is still room for better designs, particularly when the signals are critically sampled at the Nyquist rate.

Manuscript received November 22, 2010; revised February 24, 2011 and May 7, 2011; accepted June 22, 2011. Date of current version September 14, 2011. This paper was recommended by Associate Editor Y. Yu.

The author is with the Department of Electrical and Electronics Engineering, Middle East Technical University, Ankara 06531, Turkey (e-mail: ccandan@metu.edu.tr).

Color versions of one or more of the figures in this paper are available online at http://ieeexplore.ieee.org.

Digital Object Identifier 10.1109/TCSII.2011.2161176

${ }^{1}$ Throughout this brief, we assume that the continuous-time signal is sampled at the rate of 1 sample per second. The integrators discussed in this brief should be multiplied by the sampling period $T$ if $T \neq 1$.
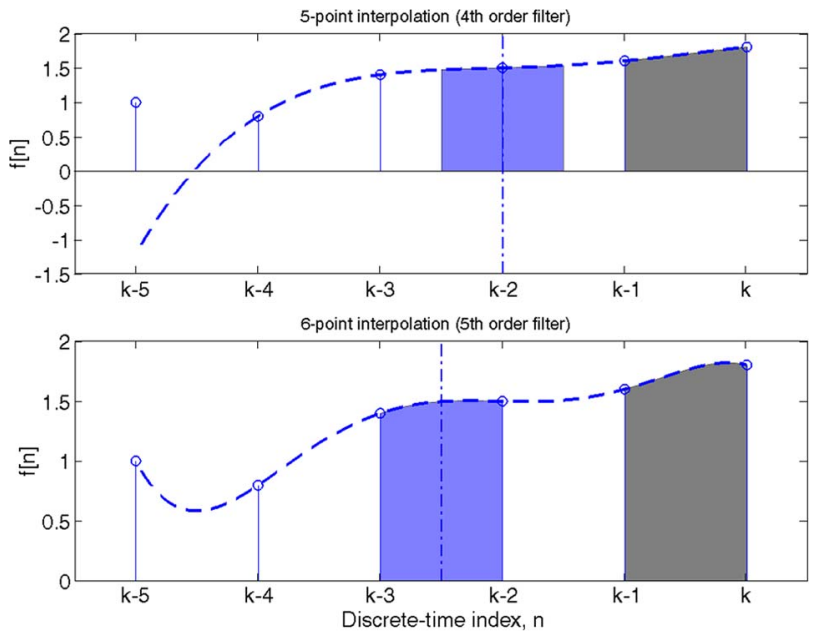

Fig. 1. Fourth- and fifth-order Lagrange interpolators for the illustration of even- and odd-order cases.

In this brief, we present a method exactly matching the phase response of the continuous-time integrator and closely approximating the desired magnitude response in a wideband. Our approach can be considered as an adaptation of the numerical integration (quadrature) rules to the integrator design.

In the literature, Ngo and Tseng have suggested the usage of quadrature rules, such as the Newton-Cotes and GaussLegendre rules, for the digital integrator design [4]-[6]. The integrators based on the Newton-Cotes rules can be expressed in the following general form [4]:

$$
H(z)=G_{N}(z) \frac{1}{1-z^{-1}} .
$$

Here, $G_{N}(z)$ is an $N$ th-order causal finite impulse response (FIR) filter. As previously discussed, the zero-order hold and the trapezoidal rule filters can be put in this form by selecting $G_{N}(z)$ appropriately [4].

The Newton-Cotes quadrature is based on finding the $N$ thorder polynomial passing through $N+1$ consecutive input samples ( $N$ th-order Lagrange interpolator) and then calculating the area under the interpolating polynomial. In the top panel of Fig. 1, an example for the fourth-order Lagrange interpolator is given. In the conventional system, the calculated area is the area between samples $(k-1)$ and $k$ (shown with gray shading) [4]. The task of the $G_{N}(z)$ filter is to execute the area calculation from the given set of $N+1$ samples. The term following $G_{N}(z)$ on the right-hand side of (1), which is $1 /\left(1-z^{-1}\right)$, is the conventional accumulator that is used to sum the area strips calculated up to that instant [4]. 
After [4], Tseng has extended and presented improved techniques using similar quadrature rules in [5] and [6]. Unfortunately, the method of Tseng requires fractional sampling rates complicating both the design and the implementation of these integrators. Tseng suggests using the Lagrange interpolators to elevate some of these problems. The most recent proposal by Tseng et al. suggests to implement the fractional delays in the Hartley transform domain [8]. In this brief, our goal is to present digital integrators using simple quadrature rules that utilize uniformly sampled data and does not require a change in sampling rate; therefore, we do not focus on fractional delays or transform domain techniques.

In this brief, our goal is to improve the performance of digital integrators as a standalone component. A reduction in the integration error can be critical for the systems in which the integration error is the predominant error source effecting the overall performance. To that aim, we present a modification on the integrators based on the Newton-Cotes rule and suggest to calculate the area of the center strip (shown with blue shading) instead of the most recent strip as done in [4]. We show that this simple modification improves the accuracy of the magnitude response significantly and leads to a perfect match of the phase response.

In [5], Tseng has presented a general theory of integrators based on the numerical integration rules and the fractional delays. It has been recognized during the review stage of this brief that the $G_{N}(z)$ polynomial of the integrators discussed here is numerically equivalent to the $U_{M}(z)$ polynomial given in [5], if $M$ and $L$ appearing in [5, eqs. (39)-(41)] are taken as $M=N$ and $L=(N-1) / 2$. To the best of our understanding, the linkage is purely coincidental (due to the choice of the Lagrange interpolation for the fractional delay implementation), and parameter $L$ appearing in [5] is considered to be an integer delay to causally implement the fractional delays. This brief can be interpreted as a novel and much simpler derivation (free of fractional delays) for a subclass of the general theory given in [5]. Furthermore, we show that the presented class of integrators surpasses previously suggested integrators including the ones in [5], which is a result not known in the literature.

This brief is organized as follows. In the following section, we explain our design motivation, then present a derivation for area calculating the $G_{N}(z)$ filter for even-odd values of $N$, and finally present a compact formula for general $N$. Then, we compare the proposed integrators with the existing proposals in the literature and conclude with further remarks.

\section{Proposed InTEgrators}

A causally implementable digital integrator in the form of (1) should a have right-sided $G_{N}(z)$. If $G_{N}\left(e^{j \omega}\right)$ is the response of a causal and linear-phase filter, then $G_{N}\left(e^{j \omega}\right)$ can be written as $G_{N}^{\mathrm{zp}}\left(e^{j \omega}\right) e^{-j(N / 2) \omega}$. Here, $N$ is the order of the filter, and $G_{N}^{\mathrm{zp}}\left(e^{j \omega}\right)$ is the zero- or constant-phase version of $G_{N}\left(e^{j \omega}\right)$. Under these conditions, the frequency response of the integrator can be written as follows:

$$
H\left(e^{j \omega}\right)=\frac{G_{N}^{\mathrm{zp}}\left(e^{j \omega}\right) e^{-j \frac{N}{2} \omega}}{e^{-j \frac{\omega}{2}}\left(e^{j \frac{\omega}{2}}-e^{-j \frac{\omega}{2}}\right)}=\frac{G_{N}^{z \mathrm{p}}\left(e^{j \omega}\right)}{2 j \sin \left(\frac{\omega}{2}\right)} e^{-j \omega(N-1) / 2} .
$$

This relation shows that a causal and linear-phase $G_{N}(z)$ can only approximate the integrator with a delay of $(N-1) / 2$ samples. It can be easily checked that, among the four types of linear-phase systems, only the type- 1 and type- 2 linear-phase systems with symmetric impulse responses $(h[n]=h[N-n])$ are suitable for the approximation of the integrator [9, p. 257].

The main idea of this brief is to impose type- 1 or type- 2 symmetry conditions on $G_{N}(z)$. To do that, we propose to calculate the area under the central strip (shown with blue shading in Fig. 1) instead of the strip conventionally calculated with the Newton-Cotes rule (shown with gray shading) [4]. This choice can be explained as follows. The blue strip contains an equal number of samples on its left and right sides. If the samples on the left and right sides are interchanged, i.e., the samples on each side are flipped to the other side, the interpolation curve also flips to the other side; however, the area under the center strip does not change. This shows that the $G_{N}(z)$ filter, which calculates the area of the center strip, should be a symmetric polynomial. In the rest of this brief, we pursue this idea and derive type- 1 and type- 2 linear-phase $G_{N}(z)$ filters. The derivation is separately given for types 1 (even $N$ ) and $2(\operatorname{odd} N)$ for the sake of clarity.

\section{A. Proposed System With Even-Order Interpolators}

We present a derivation for $G_{N}(z)$ based on the discretetime Taylor series. To introduce the discrete-time Taylor series, we first review the difference operators and the factorial polynomials. $^{2}$

The factorial polynomial $t^{[N]}$ is an $N$ th degree polynomial that is defined as $t^{[N]}=t(t+1) \ldots(t+N-1)$. The backward difference operator $\Delta$ is defined as $\Delta f[n]=f[n]-$ $f[n-1]$. When the backward difference operation is executed on the factorial polynomials, we get $\Delta t^{[N]}=N t^{[N-1]}$. Hence, with the application of the difference operator, we get a factorial polynomial with one less degree, in complete analogy with the continuous-time derivative operator and $t^{N}$ polynomial.

The discrete-time Taylor series, in analogy with its continuous version, is defined as follows:

$$
f(t)=\sum_{n=0}^{\infty} \Delta^{n} f[k] \frac{(t-k)^{[n]}}{n !} .
$$

It can be easily noted that $f(t)$ is identical to $f[k]$ when $t=k$. If $\Delta$ is applied to both sides of (3) and then $t$ is replaced with $k$, we get $\Delta f(t) \downarrow_{t=k}=\Delta f[k]$. By repeating this operation, we can show that $f(k-q)=f[k-q]$ for $q \geq 0$. This shows that $f(t)$ is an interpolating polynomial.

When the discrete-time Taylor series is truncated to a finite number of terms, the resultant relation is equivalent to fitting an $N$ th-degree polynomial to samples $\{f[k], f[k-1], \ldots, f[k-$ $(N-1)]\}$, which is the Lagrange interpolation [10].

The top panel of Fig. 1 illustrates the case for the fourth-order interpolation. We can explicitly write the relation for this case as follows:

$f(t)=f[k]+\frac{\Delta f[k]}{1 !}(t-k)+\frac{\Delta^{2} f[k]}{2 !}(t-k)^{[2]}$

${ }^{2}$ Further discussions on the Lagrange interpolation and the discrete-time Taylor series can be found in [10]. 


$$
+\frac{\Delta^{3} f[k]}{3 !}(t-k)^{[3]}+\frac{\Delta^{4} f[k]}{4 !}(t-k)^{[4]} .
$$

The Newton-Cotes formula immediately emerges when $f(t)$ given in (4) is integrated between $k-1$ and $k$. Due to the reasons explained before, we are interested in the area of the center strip, which is the integral of $f(t)$ between $k-(N+$ $1) / 2$ and $k-(N-1) / 2$, i.e.,

$$
\begin{aligned}
\int_{k-\frac{N+1}{2}}^{k-\frac{N-1}{2}} f(t) d t & =\int_{-\frac{1}{2}}^{\frac{1}{2}} f\left(t+k-\frac{N}{2}\right) d t \\
& =\sum_{n=0}^{N} \Delta^{n} f[k] \int_{-1 / 2}^{1 / 2} \frac{\left(t-\frac{N}{2}\right)^{[n]}}{n !} d t .
\end{aligned}
$$

Once the integrals are evaluated, the area under the center strip can be written as follows:

$$
\begin{aligned}
\text { Area }=f[k]+\Delta & f[k](-2)+\Delta^{2} f[k]\left(\frac{25}{24}\right) \\
& +\Delta^{3} f[k]\left(\frac{-1}{24}\right)+\Delta^{4} f[k]\left(\frac{17}{5760}\right) .
\end{aligned}
$$

When the backward difference operators are replaced with their $z$-domain counterparts, $\Delta^{L} f[k] \leftrightarrow\left(1-z^{-1}\right)^{L} F(z)$; the system function for the area calculator can be written as

$$
\begin{aligned}
G_{4}(z)= & +\left(1-z^{-1}\right)(-2)+\left(1-z^{-1}\right)^{2}\left(\frac{25}{24}\right) \\
& +\left(1-z^{-1}\right)^{3}\left(\frac{-1}{24}\right)+\left(1-z^{-1}\right)^{4}\left(\frac{17}{5760}\right) \\
= & z^{-2} \underbrace{\left(-\frac{17}{5760}\left(z^{2}+z^{-2}\right)+\frac{77}{1440}\left(z^{1}+z^{-1}\right)+\frac{863}{960}\right)}_{G_{4}^{\text {zP }}(z)} .
\end{aligned}
$$

As desired, $G_{4}(z)$ is a type-1 linear-phase sequence. A zerophase sequence, shown as $G_{4}^{\mathrm{zp}}(z)$ in the aforementioned equation, can be constructed from $G_{4}(z)$ by shifting sequence 2 samples to the left (advancing). In the general case, $G_{N}^{\mathrm{zp}}(z)$ can be constructed by advancing $G_{N}(z)$ with $N / 2$ samples.

The frequency response of the $N$ th-order integrator has the form of $H(z)=G_{N}(z) /\left(1-z^{-1}\right)$ and can be expressed as follows:

$$
H\left(e^{j \omega}\right)=\frac{G_{N}\left(e^{j \omega}\right)}{2 j \sin \left(\frac{\omega}{2}\right)} e^{j \frac{1}{2} \omega}=\frac{G_{N}^{\mathrm{zp}}\left(e^{j \omega}\right)}{2 j \sin \left(\frac{\omega}{2}\right)} e^{-j \frac{N-1}{2} \omega} .
$$

When $N$ is taken as 4 in the last relation, the resulting system is a linear-phase system with a constant group delay of $(4-1) / 2=3 / 2$ samples. In other words, the $k$ th output sample of the fourth-order integrator sums the area up to $k-(3 / 2)$ samples, which is exactly the scenario shown in the top panel of Fig. 1 .

The part of frequency response given in (8) without the linear-phase term is an approximation to the ideal integrator $\int_{-\infty}^{t} f(\tau) d \tau$. The nature of the approximation for the fourthorder case can be examined as follows:

$$
\frac{G_{4}^{\mathrm{zP}}\left(e^{j \omega}\right)}{2 j \sin \left(\frac{\omega}{2}\right)}=\frac{\frac{863}{1920}+\frac{77}{1440} \cos (\omega)-\frac{17}{5760} \cos (2 \omega)}{j \sin \left(\frac{\omega}{2}\right)}
$$

$$
\begin{aligned}
& =\frac{\frac{1}{2}-\omega^{2} \frac{1}{48}+\omega^{4} \frac{1}{3840}+\omega^{6} \frac{13}{69120}+O\left(\omega^{8}\right)}{j\left(\omega \frac{1}{2}-\omega^{3} \frac{1}{48}+\omega^{5} \frac{1}{3840}-\omega^{7} \frac{1}{645120}+O\left(\omega^{9}\right)\right)} \\
& \approx \frac{1}{j \omega} .
\end{aligned}
$$

In the second line of (9), the Taylor series expansions for the cosine and sine functions are written for the terms appearing in the numerator and the denominator. Readers should note that the numerator polynomial times $j \omega$ is equal to the denominator polynomial up to the sixth-power term. It is evident that a higher order approximation would improve the approximation accuracy by introducing more terms with identical coefficients. In the numerical comparisons section, we numerically examine the accuracy of this approximation for various values of $N$.

\section{B. Proposed System With Odd-Order Interpolators}

The bottom panel of Fig. 1 shows the Lagrange interpolator for the fifth-order interpolation and highlights the center strip. The Lagrange interpolator for the fifth-order case contains all the terms on the right-hand side of (4) and an additional term of $\left(\Delta^{5} f[k] / 5 !\right)(t-k)^{[5]}$. The area under the center strip can be calculated by integrating $f(t)$ between $k-(N+1) / 2$ and $k-(N-1) / 2$, as in the even-order case. Repeating the steps previously described, we get the type- 2 symmetric $G_{5}(z)$ polynomial as follows:

$$
\begin{array}{r}
G_{5}(z)=z^{-2.5}\left(\frac{11}{1440}\left(z^{2.5}+z^{-2.5}\right)-\frac{31}{480}\left(z^{1.5}+z^{-1.5}\right)\right. \\
\left.+\frac{401}{720}\left(z^{0.5}+z^{-0.5}\right)\right) .
\end{array}
$$

The fifth-order approximation for $1 / j \omega$ is then as follows:

$$
\begin{aligned}
& \frac{G_{4}^{\mathrm{zp}}\left(e^{j \omega}\right)}{2 j \sin \left(\frac{\omega}{2}\right)} \\
& =\frac{\frac{11}{1440} \cos (2.5 \omega)-\frac{31}{480} \cos (1.5 \omega)+\frac{401}{720} \cos (0.5 \omega)}{j \sin \left(\frac{\omega}{2}\right)} \\
& =\frac{\frac{1}{2}-\omega^{2} \frac{1}{48}+\omega^{4} \frac{1}{3840}-\omega^{6} \frac{437}{276480}+O\left(\omega^{8}\right)}{j\left(\omega \frac{1}{2}-\omega^{3} \frac{1}{48}+\omega^{5} \frac{1}{3840}-\omega^{7} \frac{1}{645120}+O\left(\omega^{9}\right)\right)} \\
& \approx \frac{1}{j \omega} .
\end{aligned}
$$

It can be seen that the frequency response expression for oddorder integrators is identical to the expression for even-order integrators given in (8). The only difference is that evenorder integrators have type-1 symmetric $G_{N}(z)$, whereas oddorder integrators have a type- 2 symmetric filter. The group delay for both cases is $(N-1) / 2$, exactly matching both illustrations given in Fig. 1.

The presented examples can be generalized to an arbitrary order without much difficulty. For an arbitrary value of $N$, the proposed digital integrators can be written as $H(z)=$ $G_{N}(z) /\left(1-z^{-1}\right)$ where

$$
G_{N}(z)=\sum_{n=0}^{N} \frac{\left(1-z^{-1}\right)^{n}}{n !} \int_{-\frac{1}{2}}^{\frac{1}{2}} \underbrace{\prod_{m=0}^{n-1}(t-N / 2+m)}_{\left(t-\frac{N}{2}\right)^{[n]}} d t .
$$


TABLE I

Proposed Digital Integrators; $H(z)=G_{N}(z) /\left(1-z^{-1}\right)$

\begin{tabular}{|c|l|}
\hline $\mathrm{N}$ & $G_{N}(z)$ \\
\hline 1 & $\frac{1}{2}+\frac{1}{2} z^{-1}$ \\
2 & $\frac{1}{24}\left(1+z^{-2}\right)+\frac{11}{12} z^{-1}$ \\
3 & $-\frac{1}{24}\left(1+z^{-3}\right)+\frac{13}{24}\left(z^{-1}+z^{-2}\right)$ \\
4 & $-\frac{17}{5760}\left(1+z^{-4}\right)+\frac{77}{1440}\left(z^{-1}+z^{-3}\right)+\frac{863}{960} z^{-2}$ \\
5 & $\frac{11}{1440}\left(1+z^{-5}\right)-\frac{31}{480}\left(z^{-1}+z^{-4}\right)+\frac{401}{720}\left(z^{-2}+z^{-3}\right)$ \\
6 & $\frac{367}{967680}\left(1+z^{-6}\right)-\frac{281}{53760}\left(z^{-1}+z^{-5}\right)+\frac{6361}{107520}\left(z^{-2}+z^{-4}\right)+\frac{215641}{241920} z^{-3}$ \\
7 & $-\frac{191}{120960}\left(1+z^{-7}\right)+\frac{1879}{120960}\left(z^{-1}+z^{-6}\right)-\frac{353}{4480}\left(z^{-2}+z^{-5}\right)+\frac{68323}{120960}\left(z^{-3}+z^{-4}\right)$ \\
\hline
\end{tabular}

Matlab Script Generating $G_{N}(z)$ Polynomials
order=5; \%set the order
syms t zi; G=1; \%init
for $\mathrm{k}=1$ :order,
$\quad$ dum=prod(t-order/2:(t-order/2+k-1))/prod(1:k);
$\quad \mathrm{G}=\mathrm{G}+\mathrm{int}(\mathrm{dum},-1 / 2,1 / 2)^{*}(1-\mathrm{zi})^{\wedge} \mathrm{k}$;
end;
simplify $(\mathrm{G})$,

The expressions for $G_{N}(z)$ up to the seventh order and the MATLAB code generating $G_{N}(z)$ for any $N$ are presented in Table I.

\section{NUMERICAL COMPARISONS AND IMPLEMENTATION ISSUES}

Here, we present a numerical comparison of the proposed integrators with the ones in the literature. The first proposal on digital integrators utilizing Newton-Cotes rules has been given in [4]. Recently, similar integrators have been proposed in [6]. The integrators proposed by Tseng have better performance when compared with other integrators requiring a similar amount of computation. We choose to compare the proposed integrators with the classical Ngo integrator, the recently proposed Tseng integrators [6], and the Simpson integrator [7]. (Interested readers can examine [6, Fig. 6] for a comparison of Tseng's integrators with the earlier integrators in the literature.)

The definitions for Ngo, Tseng, and Simpson integrators are given as follows:

$$
\begin{aligned}
& H_{N}(z) \\
& \quad=\frac{9+19 z^{-1}-5 z^{-2}+z^{-3}}{24\left(1-z^{-1}\right)} \\
& H_{T}(z) \\
& \quad=\frac{-3693+67260 z^{-1}+88650 z^{-2}-14388 z^{-3}+2139 z^{-4}}{139968\left(1-z^{-1}\right)} z \\
& H_{S}(z) \\
& \quad=\frac{1+4 z^{-1}+z^{-2}}{3\left(1-z^{-2}\right)} .
\end{aligned}
$$

Different from Ngo and Tseng integrators, the integrator based on the Simpson's rule has two poles at $z=\{1,-1\}$ and has a symmetric numerator polynomial leading to a perfect phase match with the ideal integrator. The Simpson integrator and its improved versions suggested by Tseng can be found in [7]. We note that the integrators whose poles are uniformly distributed around the unit circle, such as the Simpson integrator, can be also expressed in the framework set in [4].

Frequency Response Comparison: Fig. 2 shows the magnitude response of the proposed integrators and the ideal integrator. The next figure shows the approximation error magnitude $D\left(e^{j \omega}\right)-H\left(e^{j \omega}\right)$, which is the deviation from the desired response. Here, $D\left(e^{j \omega}\right)=1 /(j \omega)$ is the response of the ideal integrator.

For the proposed integrators presented in Figs. 2 and 3, the group delay values are different from each other. For comparison purposes, the group delay of each integrator is

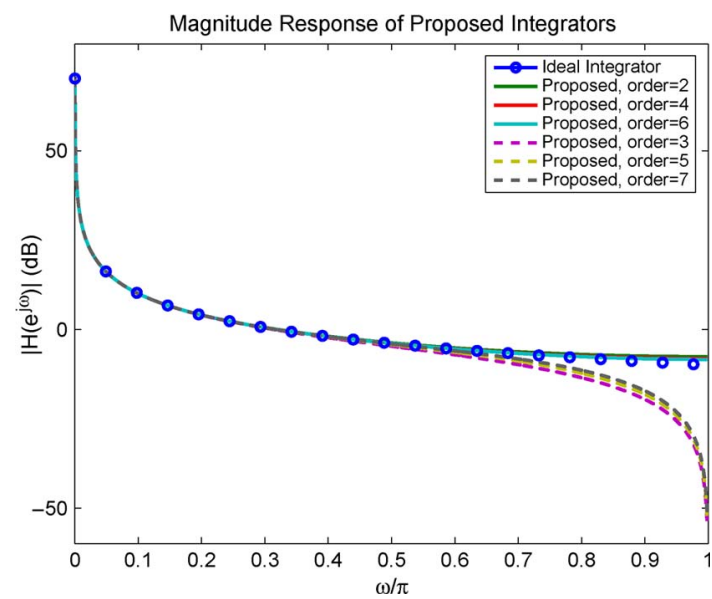

Fig. 2. Magnitude response of proposed integrators and the ideal integrator.

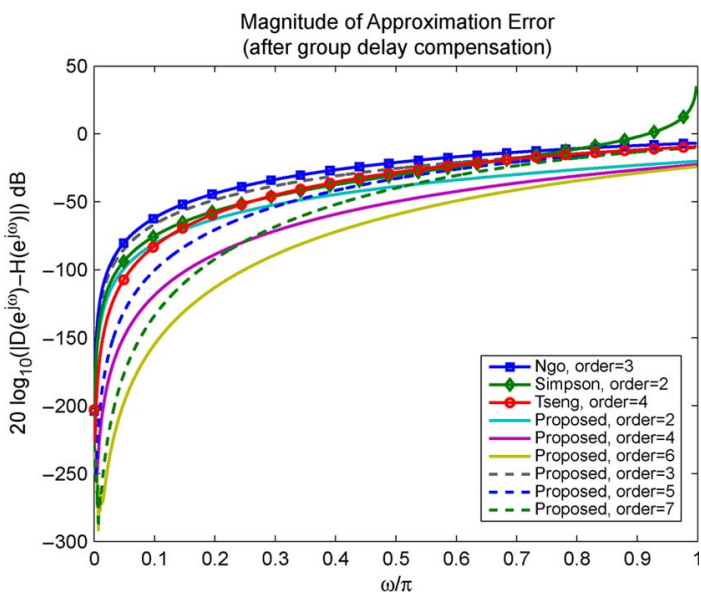

Fig. 3. Magnitude spectrum of the approximation error.

compensated, making each system a constant phase. More specifically, the linear-phase term appearing on the rightmost side of (8) is removed so that the response approximates $1 / j \omega$, as shown in (9).

It is shown in Figs. 2 and 3 that the proposed integrators, particularly the ones with even orders, perform better than earlier proposals. The proposed second-order integrator is better than the Ngo integrator (which is third order) and marginally better than the Tseng integrator (which is fourth order). The performance gap widens for the proposed fourthorder integrator.

Figs. 2 and 3 show that the integrators with even orders significantly outperform the odd-ordered ones at high frequencies. 


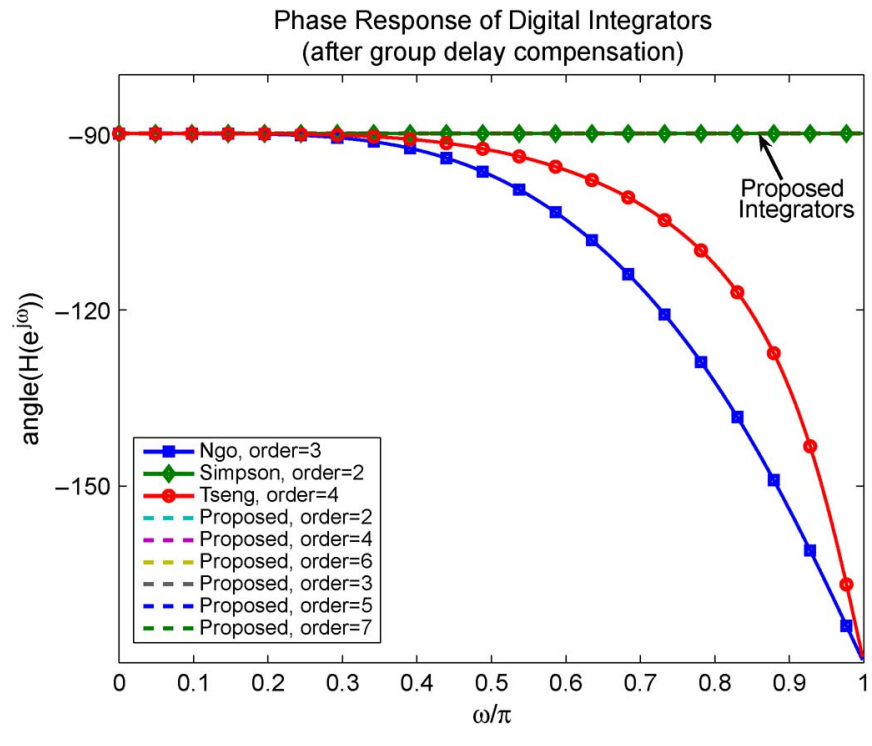

Fig. 4. Phase response of digital integrators.

This is due to the structure of type- 1 and type- 2 filters. It should be remembered that a type- 2 sequence has zero at $z=-1$, leading to $G_{2 L+1}\left(e^{j \omega}\right) \rightarrow 0$ as $\omega \rightarrow \pi$. On the other hand, the ideal integrator $1 / j \omega$ approaches $1 /(j \pi)$ as $\omega \rightarrow \pi$. The presence of zero at $z=-1$ results in an undesired attenuation of the response at high frequencies leading to an observed performance gap between even and odd orders.

Fig. 4 compares the phase response of digital integrators. The proposed integrators, by design, attain the desired phase of $-90^{\circ}$ at all frequencies. The phase of $-90^{\circ}$ is attained after the group delay compensation, which is $(N-1) / 2$ samples. For even-order integrators, this leads to a half-sample delay, as shown in the top panel of Fig. 1. It should be noted that Ngo and Tseng integrators significantly deviate from the desired response in midfrequency bands. This deviation is expected since the numerator polynomials given in the corresponding equations of (13) are not symmetric. On the other hand, the Simpson integrator has a perfect phase match due to its symmetric numerator polynomial.

Implementation Issues: The simplest digital integrator is the cumulative summer whose system function is $1 /\left(1-z^{-1}\right)$. The cumulative summer does not require any multipliers for its implementation. The proposed integrators and the other integrators with the form of $G_{N}(z) /\left(1-z^{-1}\right)$, such as the proposals of $\mathrm{Ngo}$ and Tseng, as shown in (13), require $N$ multipliers per output sample where $N$ is the order of the filter. As shown in Table $\mathrm{I}$, the proposed integrators require $\lceil(N+$ 1) $/ 2\rceil$ multipliers per output sample due to their even symmetry. (Here, $\lceil\cdot\rceil$ is the function mapping the argument to the smallest integer greater than or equal to the argument.) Therefore, there is no computational setback but a minor advantage for the proposed integrators.

It should be noted that the integrator, by definition, is an unstable system; therefore, it is only applicable for inputs having zero mean. Even for a zero-mean input, the word width of the integrator should be sufficiently large to accurately hold the accumulation result. As a final note, the two's complement (nonsaturating) arithmetic can be adopted in the implementation to aid the recovery from a potential overflow.
Application Example: To illustrate the suggested integrators, an experiment with a high-precision rate gyroscope has been conducted. In the experiment, the measurement table is programmed to make two full rotations about a single axis. The data collected with the rate gyroscope is integrated to find the instantaneous position of the object. It has been found through this experiment that the suggested integrators present an improvement in accuracy, particularly for the medium frequency inputs, i.e., $\omega \in[0.05 \pi, 0.2 \pi]$. Readers can examine the extended version of this brief for experiment details [11]. As a cautionary note, we would like to note that the error reduction due to the enhanced integrators can be insignificant in comparison with other error sources effecting the system performance in many practical applications.

\section{CONCLUSiON}

A class of digital integrators that exactly matches the phase response of the continuous-time integrator and can approximate the magnitude response of the continuous-time integrator with an arbitrary degree of accuracy across a wideband of frequencies has been given. The second- and fourth-order integrators, which require little computation per output sample, can immediately replace earlier proposals that are currently in use in many applications requiring long-term integration such as the ones in the navigation applications [1].

\section{ACKNOWLEDGMENT}

The author would like to thank the editor and the reviewers for their constructive comments and suggestions that significantly helped in improving this brief, particularly to the reviewer who has pointed the connection between the proposed integrators and the work in [5]. The author would also like to thank E. Özgeneci for his help in the construction of the described experimental setup.

\section{REFERENCES}

[1] E. Bekir, Introduction to Modern Navigation Systems. Singapore: World Scientific, 2007.

[2] B. Kumar, D. Choudhury, and A. Kumar, "On the design of linear phase, FIR integrators for midband frequencies," IEEE Trans. Signal Process., vol. 44, no. 10, pp. 2391-2395, Oct. 1996.

[3] M. Al-Alaoui, "Novel digital integrator and differentiator," Electron. Lett., vol. 29, no. 4, pp. 376-378, Feb. 1993.

[4] N. Q. Ngo, "A new approach for the design of wideband digital integrator and differentiator," IEEE Trans. Circuits Syst. II, Exp. Briefs, vol. 53, no. 9, pp. 936-940, Sep. 2006.

[5] C.-C. Tseng, "Closed-form design of digital IIR integrators using numerical integration rules and fractional sample delays," IEEE Trans. Circuits Syst. I, Reg. Papers, vol. 54, no. 3, pp. 643-655, Mar. 2007.

[6] C.-C. Tseng and S.-L. Lee, "Digital IIR integrator design using Richardson extrapolation and fractional delay," IEEE Trans. Circuits Syst. I, Reg. Papers, vol. 55, no. 8, pp. 2300-2309, Sep. 2008.

[7] C.-C. Tseng, "Digital integrator design using Simpson rule and fractional delay filter," Proc. Inst. Elect. Eng.-Vis. Image Signal Process., vol. 153, no. 1, pp. 79-86, Feb. 2006.

[8] C.-C. Tseng and S.-L. Lee, "Design of digital IIR integrator using discrete Hartley transform interpolation method," in Proc. IEEE Int. Symp. Circuits Syst., May 2009, pp. 2181-2184.

[9] A. V. Oppenheim and R. W. Schafer, Discrete-Time Signal Processing. Upper Saddle River, NJ: Prentice-Hall, 1989.

[10] C. Candan, "An efficient filtering structure for Lagrange interpolation," IEEE Signal Process. Lett., vol. 14, no. 1, pp. 17-19, Jan. 2007.

[11] C. Candan, Digital Wideband Integrators with Matching Phase and Arbitrarily Accurate Magnitude Response (Extended Version). [Online]. Available: http://www.eee.metu.edu.tr/ ccandan/pub.htm 